Pacific Journal of Mathematics

BAER AND UT-MODULES OVER DOMAINS 


\section{BAER AND UT-MODULES OVER DOMAINS}

\section{RALPH P. GRIMALDI}

For a domain $R$, an $R$-module $A$ is called a Baer module if $\operatorname{Ext}_{R}{ }_{R}^{1}(A, T)=0$ for every torsion $R$-module $T$. Dual to Baer modules, a torsion $R$-module $B$ is called a $U T$-module if $\operatorname{Ext}_{R}{ }_{R}^{1}(X, B)=0$ for every torsion free $R$-module $X$. In this paper properties of these two types of modules will be derived and characterizations of Prüfer domains, Dedekind domains and fields will be obtained in terms of Baer and UT-module properties. One characterization will show the Baer modules are analogous to projective modules in the sense that a domain $R$ is Dedekind if and only if, over $R$, submodules of Baer modules are Baer. In addition, just as a semisimple ring $S$ can be characterized by the property that all $S$-modules are injective, or, equivalently, all $S$-modules are projective, a domain $R$ is a field exactly if every torsion $R$-module is $U T$ or, equivalently, every torsion free $R$-module is a Baer module. Further properties of these two kinds of modules will provide sufficient conditions to bound the global dimension of a domain $\boldsymbol{R}$.

0. Historical Note. The concept of a Baer module goes back to 1936 when R. Baer, in [1], proposed the problem asking for a complete characterization of all abelian groups $G$ such that $\operatorname{Ext}_{Z}(G, T)=0$, for all torsion abelian groups $T$. At that time he showed that any such abelian group must be torsion free, and free if it had countable rank. Then in $1959 \mathrm{R}$. Nunke, in [10], extended these results to modules over a Dedekind domain, proving that such a module was again torsion free, and projective if it had countable rank. Finally in 1969 P. Griffith, in [5], completely solved Baer's problem for abelian groups, and showed that any such abelian group, now called a Baer group or $B$-group, must be free. In [6], the author extended Griffith's techniques to modules over a Dedekind domain, showing that if $A$ is a Baer module over a Dedekind domain then $A$ is projective. The major adjustments needed in this transition from abelian groups to modules over a Dedekind domain were accomplished by means of Corollary 2 on p. 279 of [12], Theorems 3 and 5 in [8], Lemma 8.3 and Theorem 8.4 in [10], as well as the exposition on ideals and valuations in $\$ 18,19$ of [4].

1. Preliminaries. Unless additional restrictions are stated, in this paper $R$ denotes an arbitrary integral domain: that is, a commutative ring with 1 having no zero divisors. The quotient field of $R$ will be denoted by $Q$. 
In addition, all $R$-modules will be unital $R$-modules, and all homomorphisms are $R$-homomorphisms.

For any $R$-module $A$, we define the homological dimension of $A$, denoted $h d(A)$, to be

$$
\sup \left\{n \mid \operatorname{Ext}_{R}^{n}(A, B) \neq 0, \quad \text { for some } R \text {-module } B\right\}
$$

Similarly, the injective dimension of $A$, denoted $\operatorname{id}(A)$, is defined as

$$
\sup \left\{n \mid \operatorname{Ext}_{R}^{n}(B, A) \neq 0, \quad \text { for some } R \text {-module } B\right\}
$$

Then the global dimensions of $R$, denoted $\mathrm{D}(R)$, is given by

$$
\sup \{\operatorname{hd}(A) \mid A \text { an } R \text {-module }\}
$$

we also have $\mathrm{D}(R)=\sup \{\operatorname{id}(A) \mid A$ an $R$-module $\}$, and $\mathrm{D}(R)=$ $\sup \{\operatorname{hd}(R / I) \mid I$ an ideal of $R\}$, by Theorems 9.12 and 9.14, respectively, of [11].

Replacing the functor $\operatorname{Ext}_{R}^{n}$ with $\operatorname{Tor}_{n}^{R}$, we define the flat dimension of an $R$-module $A$, denoted fd $(A)$, to be

$$
\sup \left\{n \mid \operatorname{Tor}_{n}^{R}(A, B) \neq 0, \quad \text { for some } R \text {-module } B\right\}
$$

(Since $R$ is commutative, $\operatorname{Tor}_{n}^{R}(A, B) \cong \operatorname{Tor}_{n}^{R}(B, A)$.) Arising from the flat dimension of $R$-modules we have the weak dimension of $R$, denoted by $\mathrm{wD}(R)$, and defined by $\sup \{\mathrm{fd}(A) \mid A$ an $R$-module $\}$. Theorems 9.17 and 9.19 of [11] show, respectively, that in general $\mathrm{wD}(R) \leq \mathrm{D}(R)$, while if $R$ is Noetherian, $\mathrm{wD}(R)=\mathrm{D}(R)$.

2. Baer Modules. Our first result is in part an extension of $\mathrm{Ka}$ plansky's theorem 1 in [7].

Lemma 1. Let $A$ be an $R$-module such that $\operatorname{Ext}_{R}^{n}(A, T)=0$ for every torsion $R$-module T and some fixed positive integer $n$. Then (i) $\mathrm{hd}(A) \leq n$; (ii) $\operatorname{Ext}_{R}^{n}(A, B)$ is a divisible $R$-module for any $R$-module $B$.

Proof. In proving (i), we follow Kaplansky's proof of Theorem 1 in [7] and show that for any $R$-module $C, \operatorname{Ext}_{R}^{n+1}(A, C)=0$. Given any $R$-module $C$, with $D$ its injective envelope, we get an exact sequence

$$
0 \rightarrow C \rightarrow D \rightarrow D / C \rightarrow 0
$$


with $D / C$ a torsion $R$-module, and $(*)$ gives rise to an exact sequence

$$
\operatorname{Ext}_{R}^{n}(A, D / C) \rightarrow \operatorname{Ext}_{R}^{n+1}(A, C) \rightarrow \operatorname{Ext}_{R}^{n+1}(A, D),
$$

where the first member vanishes by the hypothesis, since $D / C$ is torsion, and the third member vanishes since $D$ is injective. Hence $\operatorname{Ext}_{R}^{n+1}(A, C)=0$ and it follows that hd $(A) \leq n$.

To establish (ii), let $B$ be any $R$-module, with $t B$ its torsion submodule. For the given module $A$, the exact sequence

$$
0 \rightarrow t B \rightarrow B \rightarrow B / t B \rightarrow 0
$$

gives rise to the exact sequence

(**) $\operatorname{Ext}_{R}^{n}(A, t B) \rightarrow \operatorname{Ext}_{R}^{n}(A, B) \rightarrow \operatorname{Ext}_{R}^{n}(A, B / t B) \rightarrow \operatorname{Ext}_{R}^{n+1}(A, t B)$,

where $\operatorname{Ext}_{R}^{n}(A, t B)=0$ by the hypothesis, and $\operatorname{Ext}_{R}^{n+1}(A, t B)=0$ from (i). Consequently,

$$
\operatorname{Ext}_{R}^{n}(A, B) \cong \operatorname{Ext}_{R}^{n}(A, B / t B)
$$

so from here on we can assume that $B$ is torsion free. Then for any $0 \neq r \in$ $R, B$ torsion free implies that we get an exact sequence

$$
0 \rightarrow B \stackrel{r}{\longrightarrow} B \rightarrow B / r B \rightarrow 0
$$

which induces the exact sequence

$(* *)^{\prime \prime}$

$$
\operatorname{Ext}_{R}^{n}(A, B) \stackrel{r}{\longrightarrow} \operatorname{Ext}_{R}^{n}(A, B) \longrightarrow \operatorname{Ext}_{R}^{n}(A, B / r B)
$$

since the functor $\operatorname{Ext}_{R}^{n}(A, \quad)$ preserves multiplications. Now as $B / r B$ is torsion, $\operatorname{Ext}_{R}^{n}(A, B / r B)=0$, so every multiplication by a nonzero element of $R$ is an epimorphism, from which it follows that $\operatorname{Ext}_{R}^{n}(A, B)$ is divisible.

Definition . Over a domain $R$, an $R$-module $A$ is called a $B a e r$ module if $\operatorname{Ext}_{R}^{1}(A, T)=0$ for every torsion $R$-module $T$.

REMARKS. (1) From lemma 1 we see that if $A$ is a Baer module, then $\operatorname{Ext}_{R}^{n}(A, T)=0$ for all torsion $R$-modules $T$ and for every positive integer $n$.

(2) In [7] Kaplansky called torsion free Baer modules $U F$-modules and showed that if $A$ is a $U F$-module then $A$ is flat with $\operatorname{hd}(A) \leq 1$. However, 
the proofs of neither of these two results necessitated that $A$ be torsion free. In addition, in the proof of Theorem 4.23 in [11], the hypothesis that $R$ is a Prüfer domain, that is a domain $R$ in which every finitely generated ideal of $R$ is projective as an $R$-module, is not necessaey in proving that a flat $R$-module is torsion free, and only required that $R$ be a domain. Consequently, for any domain $R$ Baer modules are torsion free and hence equivalent to Kaplansky's $U F$-modules.

LEMMA 2. The following are satisfied for an arbitrary domain $R$ :

(i ) If $A$ is a Baer module, then every direct summand of $A$ is a Baer module.

(ii) The direct sum of a family of Baer modules is likewise a Baer module.

(iii) If $A$ is a Baer module and $P$ is a projective, then $A \otimes_{R} P$ is a Baer module.

(iv) If $0 \rightarrow A^{\prime} \rightarrow A \rightarrow A^{\prime \prime} \rightarrow 0$ is exact, with $A^{\prime}, A^{\prime \prime}$ Baer modules, then $A$ is likewise Baer.

(v) If $0 \rightarrow A^{\prime} \rightarrow A \rightarrow A^{\prime \prime} \rightarrow 0$ is exact, with $A$ a Baer module, then $A^{\prime}$ is

(vi) An R-module $A$ is Baer if and only if for every epimorphism $f: B \rightarrow$ $C$ with $\operatorname{ker} f$ torsion, $\operatorname{Hom}_{R}(A, f)$ is likewise an epimorphism. Baer if and only if $\operatorname{Ext}_{R}^{2}\left(A^{\prime \prime}, T\right) \neq 0$ for every torsion $R$-module $T$.

Proof. $\operatorname{Ext}_{R}^{1}(, T)$ commutes with direct sums, for any torsion $R$ module $T$, and so we have (i) and (ii). Then, since a projective module is a direct summand of a free module, and the tensor product commutes with direct sums, (iii) follows from (i) and (ii). The proofs of (iv) and (vi) are likewise straightforward.

Motivated by the homological definition of a Dedekind domain as one in which every ideal is projective, our next two results investigate domains in which every ideal is a Baer module.

THEOREM 3. Let $R$ be a domain in which every ideal of $R$ is a Baer module. Then $R$ is a Prüfer domain with $\mathrm{D}(R) \leq 2$.

Proof: In proving that $R$ is Prüfer all we actually need is that every ideal of $R$ is flat, which we have here by Kaplansky's theorem 2 in [7]. So for every ideal $I$ of $R$, and any $R$-module $A$, applying $A \otimes_{R}$ to the exact sequence

$$
0 \rightarrow I \rightarrow R \rightarrow R / I \rightarrow 0
$$


we get the exact sequence

$$
\operatorname{Tor}_{2}^{R}(A, R) \rightarrow \operatorname{Tor}_{2}^{R}(A, R / I) \rightarrow \operatorname{Tor}_{1}^{R}(A, I),
$$

where the end modules vanish, since $R, I$ are flat. Consequently, by Theorem 9.18 of [11] it follows that $\mathrm{wD}(R) \leq 1$, and so by Chase's Theorem 4.2 in [3], we have $R$ a Prüfer domain. Now by Lemma 2 (v) we have $\operatorname{Ext}_{R}^{2}(R / I, T)=0$ for every torsion $R$-module $T$, since $I$ is a Baer module; so by Lemma $1 \mathrm{hd}(R / I) \leq 2$, for every ideal $I$ of $R$, and it follows by an earlier statement that $\mathrm{D}(R) \leq 2$.

COROllaRY 4. If $R$ is a Noetherian domain, then $R$ is Dedekind if and only if every ideal of $R$ is a Baer module.

REMARKS. (1) If $R$ is a domain such that every ideal in $R[X]$ is a Baer module, then by Theorem 3 and the Hilbert Syzygy Theorem, $R$ must be Dedekind.

(2) If $R$ is a Dedekind domain but not a field, it follows from Corollary 4 that there exists an ideal in $R[X]$ which is not a Baer module.

(3) If $R$ is a Prüfer domain with $\mathrm{D}(R)>2$, then there is an ideal $I$ of $R$ with $\mathrm{hd}(I)>1$. Hence, although $I$ is flat, it is not a Baer module.

Restricting our interest now to the finitely generated ideals of a domain $R$, we get the following characterization of a Prüfer domain, but here again the Baer condition is more than we actually need.

TheOREM 5. A damain $R$ is Prüfer if and only if (i) the direct product of any family of flat $R$-modules is a flat $R$-module; and, (ii) every finitely generated ideal of $R$ is a Baer module.

Proof. For $R$ Prüfer, an $R$-module is torsion free if and only if it is flat, so (i) follows; (ii) follows since every finitely generated ideal is projective. Conversely, if $I$ is any finitely generated ideal in $R, I$ Baer implies $I$ flat, by (ii); by Chase's Theorem 2.1 in [3], (i) implies that $I$ is finitely related: that is, there exists a short exact sequence

$$
0 \rightarrow K \rightarrow F \rightarrow I \rightarrow 0
$$

where $F$ is free, and $K, F$ are finitely generated $R$-modules. Then by the corollary to Proposition 2.2 in [3], we get $I$ projective, and hence $R$ is Prüfer.

3. UT-Modules At this point we shall turn our attention to a module 
somewhat dual to the Baer module, and start by extending Kaplansky's Theorem 3 in [7].

LEMMA 6. If $A$ is an $R$-module such that $\operatorname{Ext}_{R}^{n}(X, A)=0$ for every torsion free $R$-module $X$, then $\mathrm{id}(A) \leq n$.

Proof. Following Kaplansky's idea in Theorem 3 in [7], we shall show that $\operatorname{Ext}_{R}^{n+1}(C, A)=0$, for any module $C$. For given any $R$-module $C$, we consider the exact sequence

$$
0 \rightarrow K \rightarrow F \rightarrow C \rightarrow 0,
$$

where $F$ is free, and $K$, consequently, torsion free. $\left({ }^{*}\right)$ then gives rise to the exact sequence

$$
\operatorname{Ext}_{R}^{n}(K, A) \rightarrow \operatorname{Ext}_{R}^{n+1}(C, A) \rightarrow \operatorname{Ext}_{R}^{n+1}(F, A),
$$

where the first module is 0 by hypothesis, while $F$ free implies the third module is 0 . Thus $\operatorname{Ext}_{R}^{n+1}(C, A)=0$ for any $R$-module $C$, and $\operatorname{id}(A) \leq n$.

Definition (Kaplansky [7]): For any domain $R$, a torsion $R$ module $A$ is called a $U T$-module if $\operatorname{Ext}_{R}^{1}(X, A)=0$ for every torsion free $R$-module $X$.

ReMARK. From Lemma 6 we see that if $A$ is a $U T$-module, then $\operatorname{Ext}_{R}^{n}(X, A)=0$ for every torsion free $R$-module $X$ and for every positive integer $n$, and $\operatorname{id}(A) \leq 1$.

Analogous to Lemma 2, we now have the following.

LEMMA 7. The following are satisfied for an arbitrary domain $R$ :

(i ) Direct summands of UT-modules are UT-modules:

(ii) Finite direct sums of UT-modules are UT-modules;

(iii) If $\left\{T_{\alpha}\right\}_{\alpha \in A}$ is a family of UT-modules, then $\operatorname{Ext}_{R}^{1}\left(X, \Pi_{\alpha \in A} T_{\alpha}\right)=$ 0 for every torsion free $R$-module $X$, and consequently, $\operatorname{id}\left(\Pi_{\alpha \in A} T_{\alpha}\right) \leq 1$. If the family $\left\{T_{\alpha}\right\}_{\alpha \in A}$ is uniformly bounded: that is, there exists $0 \neq r \in R$ such that $r T_{\alpha}=0$ for all $\alpha \in A$, then $\Pi_{\alpha \in A} T_{\alpha}$ is a bounded UT-module.

(iv) If $T$ is a UT-module and $P$ is a finitely generated projective $R$-module, then $T \otimes_{R} P$ is a UT-module.

(v) Extensions of UT-modules by UT-modules are UT-modules.

(vi) If $0 \rightarrow T^{\prime} \rightarrow T \rightarrow T^{\prime \prime} \rightarrow 0$ is an exact sequence with $T a$ $U T$-module, then $T^{\prime \prime}$ is a UT-module if and only if $\operatorname{Ext}_{R}^{2}\left(X, T^{\prime}\right)=0$ for every torsion free $R$-module $X$. 
(vii) $A$ torsion $R$-module $T$ is a UT-module if and only if for every monomorphism $f: B \rightarrow C$ with coker $f$ torsion free, $\operatorname{Hom}_{R}(f, T)$ is an epimorphism.

Proof. The proofs here are more or less dual to those for Lemma 2.

Proposition 8. If $R$ is a Prüfer domain and $T$ a bounded $R$-module, then for any injective $R$-module $Y, \operatorname{Hom}_{R}(T, Y)$ is a bounded UT-module.

Proof. Since $T$ is bounded, we get $\operatorname{Hom}_{R}(T, Y)$ bounded. By Proposition 5.1 in Chapter VI of [2],

$$
\operatorname{Ext}_{R}^{1}\left(A, \operatorname{Hom}_{R}(T, Y)\right) \cong \operatorname{Hom}_{R}\left(\operatorname{Tor}_{1}^{R}(A, T), Y\right),
$$

so if $A$ is torsion free, $R$ Prüfer implies that $\operatorname{Tor}_{1}^{R}(A, T)=0$ and the result follows.

Closely related to the concept of a $U T$-module, and in some cases equivalent to it, is the concept of a cotorsion module.

Definition (Matlis [9]). Let $R$ be a domain with quotient field $Q$. An $R$-module $C$ is called $h$-reduced if $\operatorname{Hom}_{R}(Q, C)=0$. If $C$ is h-reduced, then $C$ is called cotorsion if $\operatorname{Ext}_{R}^{1}(Q, C)=0$.

THEOREM 9. Let $R$ be a domain with quotient field $Q$, and $T$ an arbitrary torsion $R$-module. Then $R$ is Dedekind if and only if $T$ being $a$ cotorsion module is equivalent to $T$ being a reduced $U T$-module.

Proof. If $R$ is Dedekind and $T$ is torsion and cotorsion, then by Nunke's Corollary 7.8 in [10], $T$ is bounded. Also, $R$ Dedekind implies that $\operatorname{id}(T) \leq 1$, so by Kaplansky's Theorem 4 in [7], $T$ is a $U T$-module. By the definitions it follows that if $T$ is a reduced $U T$-module then $T$ is cotorsion.

Conversely, suppose that $R$ is not Dedekind, and that $T$ is any bounded torsion $R$-module. Let $0 \neq r \in R$ be a bound for $T$. Since $Q$ is torsion free, divisible, $\operatorname{Ext}_{R}^{1}(Q, T)$ is torsion free, divisible, so $\operatorname{Ext}_{R}^{1}(Q, T)$ $=r \operatorname{Ext}_{R}^{1}(Q, T)$. However, as $\operatorname{Ext}_{R}^{1}(Q, \quad)$ preserved multiplications, the homomorphism $T \stackrel{r}{\longrightarrow} T$, with $r T=0$, induces

$$
\operatorname{Ext}_{R}^{1}(Q, T) \stackrel{r}{\longrightarrow} \operatorname{Ext}_{R}^{1}(Q, T)
$$

with $0=r \operatorname{Ext}_{R}^{1}(Q, T)=\operatorname{Ext}_{R}^{1}(Q, T)$, and we have $T$ cotorsion. In fact, 
here $\operatorname{Ext}_{R}^{n}(Q, T)=0$ for all $n \geq 0$, implying that $T$ is strongly cotorsion, a concept developed by Matlis in [9]. Hence every bounded torsion $R$ module is cotorsion, but as $R$ is not Dedekind, by Chase's Theorem 4.3 in [3] there exists a bounded torsion $R$-module that is not a $U T$-module.

Now as noted previously, if $\mathrm{D}(R)>2$, then there exists an ideal $I$ of $R$ which is not a Baer module, although $I \subset R$, a Baer module. Hence it follows that in general submodules of Baer modules need not be Baer. The next theorem provides necessary and sufficient conditions for submodules of Baer to be Baer, and serves to characterize Dedekind domains

THEOREM 10. For a domain $R$, the following are equivalent:

(i ) $R$ is a Dedekind domain:

(ii) Submodules of Baer modules are Baer modules;

(iii) Submodules of projective modules are Baer modules; and,

(iv) Submodules of free modules are Baer modules.

Proof. By Lemma 2 (v), (ii) follows from (i); (iii) follows from (ii), and (iv) from (iii), since a free module is projective, which, in turn, is Baer.

In order to show that (iv) implies (i), we shall use Chase's Theorem 4.3 in [3] and show that any bounded torsion $R$-module $T$ is a UT-module. To accomplish this, by Kaplansky's Theorem 4 in [7], it is sufficient to prove that $\operatorname{id}(T) \leq 1$.

So let $A$ be any $R$-module, and consider the exact sequence

$$
0 \rightarrow K \rightarrow F \rightarrow A \rightarrow 0
$$

where $F$ is free. Then by the hypothesis, $K$ is a Baer module. The sequence $(*)$ gives rise to the exact sequence

$$
\operatorname{Ext}_{R}^{1}(K, T) \rightarrow \operatorname{Ext}_{R}^{2}(A, T) \rightarrow \operatorname{Ext}_{R}^{2}(F, T),
$$

where the first module vanishes since $K$ is Baer, and the third vanishes since $F$ is free. Consequently, $\operatorname{id}(T) \leq 1$, and it follows that $R$ is Dedekind.

4. Baer and $\boldsymbol{U}$ T-Modules. Our next theorem will show how Baer and $U T$-modules can be used in characterizing fields. The following lemma, which is Exercise 4.2 in [11], will prove useful in this characterization; we include a proof of this lemma now for the sake of completeness.

LEMMA 11. If $R$ is a domain but not a field, then an $R$-module $A$ is both injective and projective if and only if $A=0$. 
Proof. With the sufficiency clear, let us consider an $R$-module $A$ that is both projective and injective.

Since $R$ is a domain, $A$ projective implies $A$ is torsion free, and since $A$ is injective we have $A \cong \Sigma_{\alpha} \oplus Q$, where $\alpha=\operatorname{rank} A$. If $R$ is not a field, then $R$ is reduced, as is any free $R$-module, but then $A=\Sigma_{\alpha} \oplus Q$ is a direct summand of a reduced free $R$-module, since $A$ is projective. So if $0 \neq A, R$ must be a field.

THEOREM 12. For a domain $R$ with quotient field $Q$, the following are equivalent:

(i) $Q$ is a Baer and $R$ is Dedekind;

(ii) $Q$ is Baer and submodules of Baer modules are Baer;

(iii) Every torsion free R-module is Baer;

(iv) Every torsion $R$-module is a UT-module;

(v) $R=Q$ : that is, $R$ is a field;

(vi) Homomorphic images of Baer modules are Baer; and,

(vii) Submodules of UT-modules are UT.

Proof. The equivalence of (i) and (ii) follows from Theorem 10.

To prove that (ii) implies (iii), let $X$ be a torsion free $R$-module with rank $X=\alpha$. Then as $R$ is Dedekind, $X$ is flat so the inclusion $0 \rightarrow R \rightarrow Q$ induces the exact sequence

$$
0 \rightarrow X \cong R \otimes_{R} X \rightarrow Q \otimes_{R} X \cong \Sigma_{\alpha} \oplus Q,
$$

and the result follows from Lemma 2 (ii).

The equivalence of (iii) and (iv) is clear from the definitions of Baer and $U T$-modules.

In establishing (v), we first see that (iv) implies $R$ is Dedekind by Chase's Theorem 4.3 in [3]. Now by (iii), $Q$ is a Baer module, and since $R$ is Dedekind, we conclude that $Q$ is projective. Then as $Q$ is also injective, and $Q \neq 0$, it follows from Lemma 11 that $R=Q$ and $R$ is a field. Consequently, we have the equivalence of (i) - (v).

Now if $R$ is a field, every $R$-module is free, so (vi) follows from (v), and (vi) then implies (iii), since every $R$-module is the epimorphic image of a free $R$-module.

Finally, as submodules of torsion modules are torsion, (iv) implies (vii), while the converse follows because the injective envelope of a torsion $R$-module is an injective torsion module, hence a $U T$-module.

Now as a consequence of Theorem 12 we have the following two corollaries. 
Corollary 13. Let $R$ be a Dedekind domain with quotient field $Q$. Then the following are equivalent:

(i) $R=Q$ : that is, $R$ is a field;

(ii) $\operatorname{Ext}_{R}^{1}(A$,$) commutes with direct limits for every R$-module $A$;

(iii) $\operatorname{Ext}_{R}^{1}(A$,$) commutes with direct limits for every torsion free R$ module A;

(iv) $\operatorname{Ext}_{R}^{1}(Q$,$) commutes with direct limits;$

(v) Every torsion $R$-module is cotorsion; and,

(vi) Every torsion $R$-module is bounded.

Proof. With the other implications immediate from previous results, we note that (v) follows from (iv) because any torsion R-module is the direct limit of its finitely generated, hence bounded, submodules, which are consequently cotorsion modules.

Corollary 14. Let $R$ be a Prufer domain with quotient field $Q$. Then the following are equivalent:

(i) $R=Q$ : that is, $R$ is a field;

(ii) $\operatorname{Ext}_{R}^{1}(, A)$ commutes with direct limits for every $R$-module A; and,

(iii) $\operatorname{Ext}_{R}^{1}(, A)$ commutes with direct limits for every torsion $R$-module A.

Proof. Since the other implications are clear, all we need to prove is that (i) follows from (iii), and this we get from Theorem 12 since every torsion free R-module is the direct limit of its finitely generated submodules and over a Prufer domain a finitely generated torsion free Rmodule is projective.

From Theorem 10 and Lemma 7 (v), we see that if submodules of Baer modules are Baer, then the homomorphicimages of $U T$-modules are again UT-modules. We shall now prove a partial converse to this, for a certain type of submodule of a Baer module.

THEOREM 15. For any domain R, homomorphic images of UT-modules are UT-modules if and only if pure submodules of Baer modules are Baer modules.

Proof. Let

$$
0 \rightarrow A^{\prime} \rightarrow A \rightarrow A / A^{\prime} \rightarrow 0
$$

be exact with $A$ a Baer module and $A^{\prime}$ a pure submodule of $A$, so $A / A^{\prime}$ is 
torsion free. Now let $T$ be a torsion R-module and consider the exact sequence

$$
0 \rightarrow T \rightarrow D \rightarrow D / T \rightarrow 0
$$

where $D$ is the injective envelope of $T$. So $D$ is a torsion injective module and by the hypothesis $D / T$ is a UT-module.

From $\left({ }^{*}\right)$ we get the exact sequence

$$
\operatorname{Ext}_{R}^{1}(A, T) \rightarrow \operatorname{Ext}_{R}^{1}\left(A^{\prime}, T\right) \rightarrow \operatorname{Ext}_{R}^{2}\left(A / A^{\prime}, T\right) \rightarrow \operatorname{Ext}_{R}^{2}(A, T),
$$

where the end modules are 0 since $A$ is Baer, so

$$
\operatorname{Ext}_{R}^{1}\left(A^{\prime}, T\right) \cong \operatorname{Ext}_{R}^{2}\left(A / A^{\prime}, T\right)
$$

Consequently, the result will follow upon our proving that $\operatorname{Ext}_{R}^{2}\left(A / A^{\prime}, T\right)$ $=0$. So applying $\operatorname{Hom}_{R}\left(A / A^{\prime}, \quad\right)$ to $\left({ }^{* *}\right)$ we get the exact sequence

$$
\operatorname{Ext}_{R}^{1}\left(A / A^{\prime}, D / T\right) \rightarrow \operatorname{Ext}_{R}^{2}\left(A / A^{\prime}, T\right) \rightarrow \operatorname{Ext}_{R}^{2}\left(A / A^{\prime}, D\right),
$$

where the first module vanishes because $D / T$ is a UT-module with $A / A^{\prime}$ torsion free, and the third because $D$ is injective. Hence $\operatorname{Ext}_{R}^{2}\left(A / A^{\prime}, T\right)=0$ and we conclude that $A^{\prime}$ is a Baer module.

Conversely, let

$$
0 \rightarrow T \rightarrow D \rightarrow D / T \rightarrow 0
$$

be exact with $D$ a UT-module. For $X$ torsion free, consider the exact sequence

$(* *)^{\prime}$

$$
0 \rightarrow K \rightarrow F \rightarrow X \rightarrow 0
$$

where $F$ is free. $F / K \cong X$, a torsion free $R$-module, implies $K$ is pure in $F$, so by the hypothesis $K$ is a Baer module.

Applying $\operatorname{Hom}_{R}(X, \quad)$ to $\left({ }^{*}\right)^{\prime}$ gives rise to the exact sequence

$$
\operatorname{Ext}_{R}^{1}(X, D) \rightarrow \operatorname{Ext}_{R}^{1}(X, D / T) \rightarrow \operatorname{Ext}_{R}^{2}(X, T) \rightarrow \operatorname{Ext}_{R}^{2}(X, D),
$$

where the end modules are 0 since $D$ is UT and $X$ is torsion free. Hence $\operatorname{Ext}_{R}^{1}(X, D / T) \cong \operatorname{Ext}_{R}^{2}(X, T)$. Then applying $\operatorname{Hom}_{R}(, T)$ to $\left({ }^{* *}\right)^{\prime}$ we get the exact sequence 


$$
\operatorname{Ext}_{R}^{1}(K, T) \rightarrow \operatorname{Ext}_{R}^{2}(X, T) \rightarrow \operatorname{Ext}_{R}^{2}(F, T),
$$

where the end modules vanish since $K, F$ are Baer modules, so $\operatorname{Ext}_{R}^{2}(X, T)$ $=0$, and from the preceding isomorphism we conclude $\operatorname{Ext}_{R}^{1}(X, D / T)=0$ for all torsion free R-modules $X$, implying that $D / T$ is a UT-module.

REMARK. If the torsion condition is deleted in the definition of a UT-module, Theorem 15 can be restated with "Baer" replaced by "projective".

THEOREM 16. Let $R$ be a domain which satisfies one, and consequently both, of the conditions of Theorem 2.15. Then the following hold in $R$ :

(i) For every torsion free $R$-module $X, f d(X) \leq 1$, and consequently $w D(R) \leq 2$;

(ii) For any torsion free $R$-module $X$ and any torsion $R$-module $T$, $\operatorname{Ext}_{R}^{n}(X, T)=0$ for all $n \geq 2$, and consequently $D(R) \leq 3$; and,

(iii) For any $R$-module $A$ and any divisible $R$-module $B$,

$$
\operatorname{Tor}_{1}^{R}(B, A / t A) \cong \operatorname{Tor}_{1}^{R}(B, A) / \operatorname{Tor}_{1}^{R}(B, t A) .
$$

Proof. Considering the exact sequence

$$
0 \rightarrow K \rightarrow F \rightarrow X \rightarrow 0
$$

where $F$ is free, if $X$ is torsion free, then $K$ is pure in $F$, so by our hypothesis $K$ is Baer, and consequently flat. Therefore, for any $R$-module $A,\left({ }^{*}\right)$ induces the exact sequence

$$
\operatorname{Tor}_{2}^{R}(F, A) \rightarrow \operatorname{Tor}_{2}^{R}(X, A) \rightarrow \operatorname{Tor}_{1}^{R}(K, A),
$$

where the end modules vanish since $F, K$ are flat. Hence $f d(X) \leq 1$, and as every ideal $I$ of $R$ is torsion free,

$$
\mathrm{wD}(R)=\sup \{f d(R / I) \mid I \text { an ideal of } R\} \leq 2
$$

To establish (ii), let $T$ be a torsion $R$-module and consider the exact sequence

$$
0 \rightarrow T \rightarrow D \rightarrow D / T \rightarrow 0
$$

where $D$ is the injective envelope of $T$, so $D / T$ is UT by the hypothesis. For 
any torsion free $X$, applying $\operatorname{Hom}_{R}(X$,$) to \left({ }^{* *}\right)$ induces the exact sequence

$$
\operatorname{Ext}_{R}^{n-1}(X, D / T) \rightarrow \operatorname{Ext}_{R}^{n}(X, T) \rightarrow \operatorname{Ext}_{R}^{n}(X, D),
$$

where the end modules vanish for $n \geq 2$ since $D, D / T$ are UT-modules. Hence by Lemma 1 (i), $h d(X) \leq 2$, and since every ideal is torsion free,

$$
D(R)=\sup \{h d(\dot{R} / I) \mid I \text { an ideal of } R\} \leq 3 .
$$

Finally, for (iii), if $A$ is any $R$-module, and $B$ a divisible $R$-module, the exact sequence

$$
0 \rightarrow t A \rightarrow A \rightarrow A / t A \rightarrow 0
$$

induces the exact sequence

$\operatorname{Tor}_{2}^{R}(B, A / t A) \rightarrow \operatorname{Tor}_{1}^{R}(B, t A) \rightarrow \operatorname{Tor}_{1}^{R}(B, A) \rightarrow \operatorname{Tor}_{1}^{R}(B, A / t A) \rightarrow B \otimes_{R} t A$

where the first module is 0 by (i) and the last is 0 since $B$ is divisible and $t A$ is torsion. The desired isomorphism then follows from the resulting short exact sequence.

We now close with some remarks on analogous ideas for Tor.

Remarks. (1) For any $R$-module $A$, if $\operatorname{Tor}_{1}^{R}(A, T)=0$ for every torsion $R$-module $T$, then $\operatorname{Tor}_{1}^{R}(A, R / I)=0$ for every ideal $I$ of $R$, and we have $A$ flat, and consequently torsion free.

(2) If $A$ is any $R$-module such that $\operatorname{Tor}_{1}^{R}(A, X)=0$ for every torsion free $R$-module $X$, by applying $A \otimes_{R}$ to the exact sequence

$$
0 \rightarrow I \rightarrow R \rightarrow R / I \rightarrow 0
$$

for $I$ an ideal of $R$, we conclude that $\operatorname{Tor}_{2}^{R}(A, R / I)=0$, so $f d(A) \leq 1$. We also note that $A$ need not be flat, for the torsion module $Q / R$ satisfies $\operatorname{Tor}_{1}^{R}(Q / R, X)=0$ for every torsion free $R$-module $X$, so $f d(Q / R)=1$.

\section{REFERENCES}

1. R. Baer, The subgroup of the elements of finite order of an Abelian group, Annals Math. 37 (1936), 766-781.

2. H. Cartan, and S. Eilenberg, Homological Algebra, Princeton University Press, Princeton (1956). 
3. S. Chase, Direct products of modules. Trans. Amer. Math. Soc., 97 (1960), 457-473.

4. C. Curtis, and I. Reiner, Representation Theory of Finite Groups and Associative Algebras, Interscience Publishers, New York (1962).

5. P. Griffith, $A$ solution to the splitting mixed group problem of Baer, Trans. Amer. Math. Soc., 139 (1969), 261-269.

6. R. Grimaldi, Baer and UT-Modules over Domains, Ph.D. thesis, New Mexico State University, 1972.

7. I. Kaplansky, The splitting of modules over integral domains, Arch. Math., 13 (1962), 341-343.

8. G., Kolettis, Homogeneous decomposable modules, Studies on Abelian Groups, Symposium, Montpellier (1967), 223-238.

9. E. Matlis, Cotorsion modules, Mem. Amer. Math. Soc., 49 (1964).

10. R. Nunke, Modules of extensions over Dedekind rings, Illinois J. Math., 3 (1959), 222-241.

11. J. Rotman, Notes on Homological Algebra, Van Nostrand Reinhold Company, New York (1970).

12. P. Samuel, and O. Zariski, Commutative Algebra, Vol. 1., Van Nostrand, Princeton (1958).

Received July 20, 1972 and in revised form January 14, 1974.

New Mexico State University 


\section{PACIFIC JOURNAL OF MATHEMATICS}

\section{EDITORS}

RICHARD ARENS (Managing Editor)

University of California

Los Angeles, California, 90024

\section{R. A. BEAUMONT}

University of Washington

Seattle, Washington 98105

\section{J. DugunDJI}

Department of Mathematics University of Southern California Los Angeles, California 90007

D. Gillbarg AND J. MilgRAM

Stanford University Stanford, California 94305

\section{ASSOCIATE EDITORS}

E. F. BECKENBACH

B. H. NEUMANN

SUPPORTING

UNIVERSITY OF BRITISH COLUMBIA

UNIVERSITY OF CALIFORNIA

MONTANA STATE UNIVERSITY

UNIVERSITY OF NEVADA

NEW MEXICO STATE UNIVERSITY

OREGON STATE UNIVERSITY

UNIVERSITY OF OREGON OSAKA UNIVERSITY
F. WOLF

K. YosHIDA

INSTITUTIONS

UNIVERSITY OF SOUTHERN CALIFORNIA

STANFORD UNIVERSITY

UNIVERSITY OF TOKYO

UNIVERSITY OF UTAH

WASHINGTON STATE UNIVERSITY

UNIVERSITY OF WASHINGTON

AMERICAN MATHEMATICAL SOCIETY

NAVAL WEAPONS CENTER

The Supporting Institutions listed above contribute to the cost of publication of this Journal, but they are not owners or publishers and have no responsibility for its content or policies.

Mathematical papers intended for publication in the Pacific Journal of Mathematics should be in typed form or offset-reproduced, (not dittoed), double spaced with large margins. Underline Greek letters in red, German in green, and script in blue. The first paragraph or two must be capable of being used separately as a synopsis of the entire paper. Items of the bibliography should not be cited there unless absolutely necessary, in which case they must be identified by author and Journal, rather than by item number. Manuscripts, in duplicate if possible, may be sent to any one of the five editors. Please classify according to the scheme of Math. Rev. Index to Vol. ${ }^{39}$. All other communications to the editors should be addressed to the managing editor, or Elaine Barth, University of California, Los Angeles, California, 90024.

100 reprints are provided free for each article, only if page charges have been substantially paid. Additional copies may be obtained at cost in multiples of 50 .

The Pacific Journal of Mathematics is issued monthly as of January 1966. Regular subscription rate: $\$ 72.00$ a year (6 Vols., 12 issues). Special rate: $\$ 36.00$ a year to individual members of supporting institutions.

Subscriptions, orders for back numbers, and changes of address should be sent to Pacific Journal of Mathematics, 103 Highland Boulevard, Berkeley, California 90708.

\section{PUBLISHED BY PACIFIC JOURNAL OF MATHEMATICS, A NON-PROFIT CORPORATION}

Copyright (C) 1974 by Pacific Journal of Mathematics

Manufactured and first issued in the U.S.A. 


\section{Pacific Journal of Mathematics}

\section{Vol. 54, No. $2 \quad$ June, 1974}

John Edward Coury, Walsh series with coefficients tending monotonically to

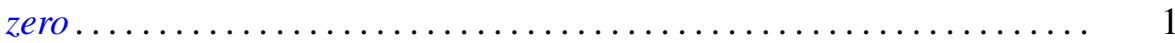

Patrick Michael Fitzpatrick and Walter Volodymyr Petryshyn, Fixed point theorems for multivalued noncompact acyclic mappings ............

Irving Leonard Glicksberg, More on Phragmén-Lindelöf for function

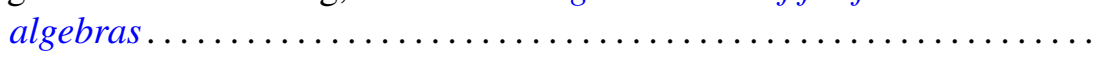

Adilson Goncalves, Structural constants. II .................. 39

Richard P. Gosselin, Closure theorems for affine transformation groups .... 53

Ralph Peter Grimaldi, Baer and UT-modules over domains ........... 59

Edward Grossman, On the prime ideal divisors of $\left(a^{n}-b^{n}\right) \ldots \ldots \ldots \ldots . \ldots 73$

A. Hedayat and Ester Seiden, On the theory and application of sum composition of Latin squares and orthogonal Latin squares.......... .

Gerald L. Itzkowitz, Continuous measures, Baire category, and uniform continuity in topological groups ......................... 115

Francis Masat, Right simple congruences on a semigroup ............ 127

Robert Harvey Oehmke, Right congruences and semisimplicity for Rees matrix semigroups..................................

Qazi Ibadur Rahman and Jan Stankiewicz, Differential inequalities and local valency . . . . . . . . . . . . . . . . . . . . . . . . . . . . . . . . . . . . . 165

William John Reed, Random points in a simplex ................ 183

Mohan S. Shrikhande, Strongly regular graphs and group divisible

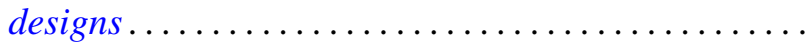

Zahava Shmuely, The structure of Galois connections ... . .

Robert C. Shock, Dual generalizations of the Artinian and Noetherian

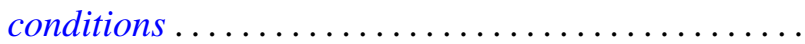

Arne Stray, Approximation and interpolation for some spaces of analytic functions in the unit disc ........................

Eldon Jon Vought, Monotone decompositions into trees of Hausdorff continua irreducible about a finite subset ............

James Wirth, The mapping cylinder axiom for WCHP fibrations ... 263

Gordon S. Woodward, Invariant means and ergodic sets in Fourier analysis... 